\title{
The Topological Structure of Microwave Circuit Theory
}

\author{
Said Mikki
}

\begin{abstract}
We present a general theory of linear microwave circuits based on continuous (Lie) groups. It is shown that the fundamental relationship between the low- and highfrequency circuits can be fully understood only using the machinery of Lie theory. By identifying classes of microwave circuit with matrix (Lie) groups, we managed to derive the most general differential equation of $n$-port network, in which the low-frequency circuit turns out to be the associated Lie algebra. The solution of the equation is formally expressed in terms of ordered exponential operators.
\end{abstract}

Index Terms-Microwave network theory, nonuniform transmission line, Lie groups.

\section{INTRODUCTION}

A microwave circuit is essentially a transmission line (TL), which in turn is a distributed-element circuit. In this view, a TL is understood as a high-frequency circuit that behaves "globally" in a very different manner compared to the low-frequency counterpart, which represents the local structure of the system. In particular, only an infinitesimal (electrically small) section of a TL looks like a lumpedelement circuit. This immediately suggests to us the analogy with differentiable (topological) manifolds, which by definition look locally like Euclidian spaces. Indeed, the universe of lumped-element circuit theory is taken as the point of departure for any circuit theory. We know very well how to do things in the low-frequency regime. Subsequently, the behavior of the global system, i.e., the full high-frequency circuit, is constructed in the following way. We start with an initial small section of a TL. Cascade another small section. The resulting system is larger than the initial one, but sill lying in its "neighborhood" (a topological concept). The process is iterated by inserting new infinitesimal sections till the global (full) picture of the complete high-frequency system is obtained. In this case, any microwave circuit can be described in terms of repeated application of infinitesimal transformations, acting in an abstract differentiable (and hence topological) space. The most natural mathematical device to handle this problem is the theory of Lie groups [2], which is well developed for classical (matrix) groups. It is a happy coincidence that microwave networks are described by (invertible) square matrices, which motivates direct application of topology and group theory to the traditional area of microwave engineering. This we endeavor to achieve in the present paper.

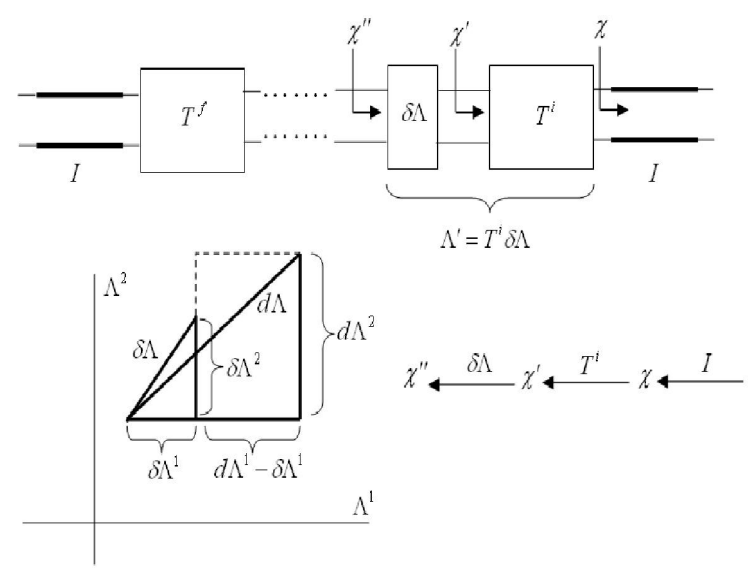

Fig. 1. Derivation of the master equation.

\section{DeVelopment of The TheORY}

\section{A. Derivation of the Master Differential Equation}

Let $G$ be a Lie group ${ }^{1}$ of dimension $M$ and the composition operation are analytic

$$
\chi^{\prime m}=\phi^{m}\left(\Upsilon^{m}, \chi^{m}\right), m=1,2, \ldots, M,
$$

In the case of $n$ input/output ports microwave circuits, the largest possible $G$ is the general linear group $\operatorname{GL}(2 n ; \mathbb{C}){ }^{2}$ Each point $g \in G$ in the differential manifold of the group will correspond to a microwave network. Here one may think of the arrays of numbers $\chi^{\prime m}, \chi^{m}$, and $\Upsilon^{m}$ as coordinates chosen in a coordinate patch containing the point (group element) $g$ in the manifold $G$. It is understood that the representation of this group element is the usual chain matrix and the group operation (1) is the usual matrix multiplication.

Now, consider Figure 1. The infinitesimal circuits connecting $T_{i}$ and $T_{f}$ are described by the matrices $\delta \Lambda$ defined along the line, and $\chi$ is the total attained chain matrix while progressing with the infinitesimally continued steps $\delta \Lambda$. We begin with the chain matrix $\chi$ seen after the reference (identity) line. The initial network $T^{i}$ will

\footnotetext{
${ }^{1}$ A Lie group is defined as a differential manifold equipped with a group operation such that the multiplication of two elements and the inverse are smooth. A matrix group is a Lie group [2].

${ }^{2}$ This is the group of square $2 n \times 2 n$ invertible matrices over the field $\mathbb{C}$ of complex numbers (we assume time-harmonic excitation throughout the paper.) Interesting physically realizable microwave circuits are matrix subgroups of $\mathrm{GL}(2 n ; \mathbb{C}$ ) (and hence Lie groups) for reasons to be given elsewhere.
} 
transform this value into $\chi^{\prime}$. The continuation process is started by inserting a small section with a chain matrix $\delta \Lambda$, producing in turn $\chi^{\prime \prime}$. We calculate $d \chi^{\prime m}=\chi^{\prime \prime m}-\chi^{m}$ using the group operation functions (1); this can be written as $\chi^{\prime \prime m}=\chi^{\prime m}+d \chi^{\prime m}=\phi^{m}\left(\delta \Lambda, \chi^{\prime}\right)$. Expanding the functions $\phi^{m}$ in Taylor series with respect to the first argument, we find to the first order

$$
d \chi^{\prime m}=\left.\sum_{n=1}^{M} \delta \Lambda^{n} \frac{\partial}{\partial \Upsilon^{n}} \phi^{m}\left(\Upsilon, \chi^{\prime}\right)\right|_{\Upsilon=0} .
$$

On the other hand, we can compute the same quantity by treating the inserted section $\delta \Lambda$ as the germ of new analytical continuation of $\chi .^{3}$ To see this, notice that $\Lambda^{\prime m}=\Lambda^{m}+d \Lambda^{m}=\phi^{m}(\delta \Lambda, \Lambda)$, where here $\Lambda^{\prime}$ can be interpreted as the new chain matrix obtained by cascading $T^{i}$ and then $\delta \Lambda$, i.e., $\Lambda^{\prime}=\delta \Lambda T^{i}$. By again expanding in Taylor series, we obtain to the first order

$$
d \Lambda^{m}=\left.\sum_{n=1}^{M} \delta \Lambda^{n} \frac{\partial}{\partial \Upsilon^{n}} \phi^{m}(\Upsilon, \Lambda)\right|_{\Upsilon=0} .
$$

Now, define the following matrix

$$
U_{n}^{-1, m}(\Lambda):=\left.\frac{\partial}{\partial \Upsilon^{n}} \phi^{m}(\Upsilon, \Lambda)\right|_{\Upsilon=0}
$$

Therefore, (3) can be written as $\delta \Lambda^{m}=\sum_{n=1}^{M} U_{n}^{m} d \Lambda^{n}$. Substituting this into (2), we obtain

$$
d \chi^{\prime m}=\sum_{n=1}^{M} \sum_{n^{\prime}=1}^{M} d \Lambda^{n^{\prime}} U_{n^{\prime}}^{n}(\Lambda) V_{n}^{m}\left(\chi^{\prime}\right),
$$

where the matrix $V$ is defined as

$$
V_{n}^{m}\left(\chi^{\prime}\right):=\left.\frac{\partial}{\partial \Upsilon^{n}} \phi^{m}\left(\Upsilon, \chi^{\prime}\right)\right|_{\Upsilon=0} .
$$

Differentiating (5) and carefully labeling the indices, it easily follows that

$$
\frac{\partial \chi^{\prime m}}{\partial \Lambda^{n}}=\sum_{r=1}^{M} U_{n}^{r}(\Lambda) V_{r}^{m}\left(\chi^{\prime}\right)
$$

The key observation in the previous derivation is that, generally speaking, $\delta \Lambda$ and $d \Lambda$ are different quantities. We can freely vary $\delta \Lambda$, which represents the inserted infinitesimal section of the tapered line; however, we can not control $d \Lambda$, or equivalently $d \chi$, which are governed by the particular Lie group structure encapsulated in the functions (1).

We develop now the problem in terms of one parameter $l$, which serves as the longitudinal index of the TL. We can express the response of the network at the location $l$ as $\chi^{m}(l)=\sum_{n=1}^{M} P_{n}^{m}(l) \chi^{n}(0)$, where we define $P$ as the one-parameter propagator of the network. Using the

\footnotetext{
${ }^{3}$ This is possible because both the new cascaded section's chain matrix and the law upon which we calculate the new value of $\chi$ are the same, i.e, matrix multiplication.
}

chain rule, the total derivative of the response $\chi$ can be computed as

$$
\frac{d}{d l} \chi^{m}(l)=\sum_{n=1}^{M} \frac{\partial \chi^{m}(l)}{\partial \Lambda^{n}} \frac{d \Lambda^{n}(l)}{d l} .
$$

Substituting (7) into (8), we obtain

$$
\frac{d}{d l} \chi^{m}(l)=\sum_{n=1}^{M} \sum_{r=1}^{M} U_{n}^{r}(\Lambda) V_{r}^{m}(\chi) \frac{d \Lambda^{n}(l)}{d l} .
$$

The infinitesimal generators of the Lie group are defined as [2]

$$
X_{n}(\chi):=-\sum_{m=1}^{M} V_{n}^{m}(\chi) \frac{\partial}{\partial \chi^{m}}
$$

Therefore, equation (9) can be put in the convenient form ${ }^{4}$

$$
\begin{aligned}
& \frac{d}{d l} P_{n}^{m}(l)=-\sum_{v=1}^{M} \sum_{r=1}^{M} \frac{d \Lambda^{v}(l)}{d l} U_{v}^{r}[\Lambda(l)] \\
& \quad \times X_{r}\left[\sum_{r=1}^{M} P_{r}^{1}(l) \chi^{r}(0), \ldots, \sum_{r=1}^{M} P_{r}^{M}(l) \chi^{r}(0)\right] P_{n}^{m}(l) .
\end{aligned}
$$

This is a system of first-order ordinary differential equations with the initial condition $P_{n}^{m}(l=0)=\delta_{n}^{m}$. It is the dynamic equation governing the propagation of electromagnetic signals along the $n$-port TL, and appears to be derived here for the first time in this context. It can be shown that (11) reduces to the familiar TL model using KCL/KVL analysis of RLC infinitesimal sections. However, (11) was derived in a systematic manner for arbitrary $n$-port network without any special assumption about the physical realization of the low-frequency element. The physical content is injected into (11) through the per-unit length parameters $d \Lambda^{v}(l) / d l$. However, the essential content of the relationship between the low- and high- frequency circuits is encoded in the Lie algebra (see next section), or the algebraic structure of the infinitesimal generators $X_{i}$. Our equation (11) clearly relates these generators to the formal solution, although in a rather complicated way. In particular, the coupling between multiple ports (e.g., chain matrices in $\operatorname{GL}(2 n ; \mathbb{C}), n>1$ ) at the lowfrequency level will be reflected into the rich mathematical structure of the Lie algebra, presenting the appropriate form of the internal configuration of microwave circuits. ${ }^{5}$

\section{B. The Lie Algebra of Microwave Circuits}

Lie agebra ${ }^{6}$ represents a linearization of the Lie group, or a local viewpoint. Surprisingly, it turns out that one can learn much about the global behavior of the group from the structure of the Lie algebra [2], [3]. In order

\footnotetext{
${ }^{4}$ To prove this, just substitute (10) to (9) and use the relation $\delta_{n}^{m}=$ $\partial\left(x^{m}\right) / \partial x^{n}$ and the definition of the circuit propagator $P$.

${ }^{5}$ The expanded version of the present paper will pay detailed considerations to some of these subtle issues.

${ }^{6}$ The Lie algebra is the vector space spanned by the infinitesimal generators $X_{i}$ closed under the commutation operation $\left[X_{i}, X_{k}\right]:=$ $X_{i} X_{k}-X_{k} X_{i}$ and satisfying the Jacobi identity [2].
} 
to motivate the concept of infinitesimal generator, it will be easier to work directly with a matrix group $\Lambda$ than the original (more general) definition (10). Expanding in Taylor series, we find $\Lambda=I+\sum_{i=1}^{M} \delta \Lambda^{i} X_{i}+O\left(\delta \Lambda^{2}\right)$, where the infinitesimal matrix generators $X_{i}$ are defined by $X_{i}:=\partial \Lambda\left(\Lambda^{1}, \ldots, \Lambda^{M}\right) / \partial \Lambda^{i}$. Here we notice that the parameters $\Lambda^{m}$ are chosen such that $\Lambda(0)=I$ and the derivatives are calculated at $\Lambda^{i}=0$. To the first order, we approximate an infinitesimal section of a TL by the matrix $\delta \Lambda \approx I+\sum_{i=1}^{M} \delta \Lambda^{i} X_{i}$. Each infinitesimal network is the low-frequency (lumped-element) circuit with perunit length parameters $\delta \Lambda$; the effective chain matrix of the TL is the multiplication of all these small sections. Therefore, by carrying out the formal limit, one can use this method to systematically compute the chain matrix of a uniform TL using the matrix exponential [2]

$$
\Lambda=\exp \left(\sum_{i=1}^{M} \xi^{i} X_{i}\right) .
$$

Concrete calculations with familiar uniform TLs using (12) will be given elsewhere. The generalization to nonuniform TLs is the subject of the next section.

\section{General Solution of Generalized Nonuniform Transmis- sion Line}

Start by defining

$$
-\frac{i}{\hbar} \mathcal{H}(l):=-\sum_{v=1}^{M} \sum_{r=1}^{M} \frac{d \Lambda^{v}(l)}{d l} U_{v}^{r}(\Lambda) X_{r}(\chi),
$$

where $\mathcal{H}$ plays the role of the Hamiltonian operator in quantum field theory and $\hbar$ the Planck constant. The master equations assumes then the following compact form

$$
\frac{d}{d l} P_{n}^{m}(l)=-\frac{i}{\hbar} \mathcal{H}(l) P_{n}^{m}(l) .
$$

Integrating (14), we obtain the following integral equation

$$
P_{n}^{m}(l)=P_{n}^{m}(0)-\int_{0}^{l} d l^{\prime} \frac{i}{\hbar} H\left(l^{\prime}\right) P_{n}^{m}\left(l^{\prime}\right),
$$

which can be viewed as the first-order iteration. Reiterating, we obtain

$$
\begin{aligned}
& P_{n}^{m}(l)=P_{n}^{m}(0)-P_{n}^{m}(0) \frac{i}{\hbar} \int_{0}^{l} d l^{\prime} H\left(l^{\prime}\right) P_{n}^{m}\left(l^{\prime}\right) \\
& \quad+\left(\frac{i}{\hbar}\right)^{2} \int_{0}^{l} d l^{\prime} H\left(l^{\prime}\right) \int_{0}^{l^{\prime}} d l^{\prime \prime} H\left(l^{\prime \prime}\right) P_{n}^{m}\left(l^{\prime \prime}\right)+\cdots
\end{aligned}
$$

The final expression can be written compactly as

$$
P_{n}^{m}(l)=\tau \exp \left[-\frac{i}{\hbar} \int_{0}^{l} d l^{\prime} \mathcal{H}\left(l^{\prime}\right)\right]
$$

where $\tau$ is the ordered exponential operator [3]. To our knowledge, the TL's propagator (17) is derived here for the first time.

Fig. 2 illustrates the space of the Lie algebra of a generalized TL problem. Two nonuniform TLs are represented by pathes I and II. The particular shape of the

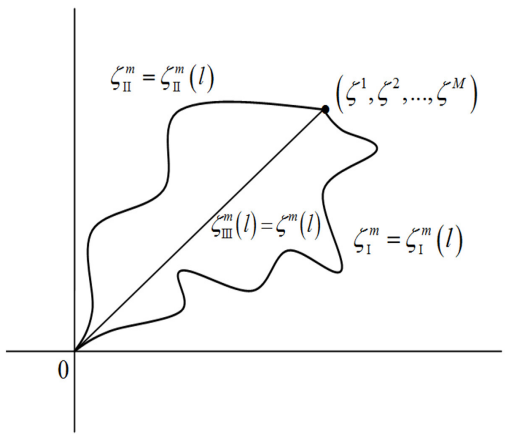

Fig. 2. Various trajectories in the Lie algebra of microwave networks.

path reflects how the low-frequency circuit is changing while progressing along the TL. Path III models a uniform TL and can be computed by the exponential map (12). From Lie theory, a neighborhood of the identity 0 in the Lie algebra will be mapped injectively into the connected identity competent of a Lie group having the same Lie algebra. As it turns out, the exponential map is not always onto, which provides a significant insight into the relation between high- and low- frequency circuits. In general, our strategy will be to start from a given Lie algebra, i.e., a low-frequency description, and then study the structure of the microwave network, i.e., the high-frequency circuit, generated by the exponential relation.

\section{Further Development of the Theory}

It can be shown that both reciprocal and lossless networks form matrix (Lie) subgroups of the general linear group. By studying the group's topology of these and other classes, we obtain general theorems about pathconnectedness, which is translated into the possibility of performing continuous matching (taper design.) On the other hand, by applying techniques from linear algebra to analyze the structure of the corresponding Lie algebras, it will become possible to decide which microwave circuits can be represented by uniform TLs, a cascade connections of two uniform TLs, etc. Also, new light will be shed on the analysis and design of nonuniform TLs by exploiting the logic of the interrelation between Lie subalgebras and Lie subgroups.

\section{CONCLUSION}

The theory developed above allows conducting thorough investigations of the fundamental aspects of how lowand high- frequency phenomena in electromagnetic wave propagation, examined here within the specialized context of circuit theory, are structurally interrelated to each other. We anticipate that the theory will provide a rigorous conceptual framework for current and future microwave circuit research. 


\section{REFERENCES}

[1] Robert E. Collin, Foundations of Microwave Circuit Theory, WileyIEEE Press, 2 edition, 2000.

[2] P. M. Cohn, Lie Groups, Cambridge University Press, 1957.

[3] E. Zeidler, Quantum Field Theory I, Springer, 2011.

[4] Said Mikki and Yahia Antar, New Foundations for Applied Electromagnetics, Artech House, 2016.

[5] S. Mikki and Y. M. M. Antar, "A topological approach for the analysis of the structure of electromagnetic flow in the antenna near-field zone," 2013 IEEE Antennas and Propagation Society International Symposium (APSURSI), Orlando, FL, 2013, pp. 17721773. 